\title{
REATIVIDADE EM REAÇÕES DE DIELS-ALDER: UMA PRÁTICA COMPUTACIONAL
}

\section{Valdemar Lacerda Júnior*}

Departamento de Química, Centro de Ciências Exatas, Universidade Federal do Espírito Santo, Av. Fernando Ferrari, 514, 29060-900 Vitória - ES, Brasil

Kleber Thiago de Oliveira, Rodrigo Costa e Silva, Mauricio Gomes Constantino e Gil Valdo José da Silva

Departamento de Química, Faculdade de Filosofia, Ciências e Letras de Ribeirão Preto, Universidade de São Paulo, Av. Bandeirantes, 3900, 14040-901 Ribeirão Preto - SP, Brasil

Recebido em 4/4/06; aceito em 14/6/06; publicado na web em 19/1/07

\begin{abstract}
REACTIVITY IN DIELS-ALDER REACTIONS: A COMPUTATIONAL EXPERIMENT. A computational quantum chemistry experiment is described of Diels-Alder reactions between 2-cycloenones and cyclopentadiene. The effects of FMO-Frontier Molecular Orbitals (HOMO$L U M O$ ) and of the withdrawing nature of substituents at the $\mathrm{C}=\mathrm{C}$ bond of cycloenones were evaluated. The calculations were made using HF/STO-3G and B3LYP/6-31+G(d,p) methods. The FMO based indexes are in agreement with the experimentally observed reactivity order. NBO - Natural Bond Orbitals - analysis was used to ascertain the effect of $\mathrm{C}=\mathrm{C}$ substituents on the dienophile reactivity.
\end{abstract}

Keywords: Diels-Alder reactivity; FMO theory; NBO analysis.

\section{INTRODUÇÃO}

A aliança Ciência e Tecnologia vem provocando nas últimas décadas várias transformações que implicam no desenvolvimento de ambas. Assim, a tecnologia não pode passar despercebida por um setor bastante relevante da nossa vida cotidiana: a Educação. Especialmente em Química, existe uma grande dificuldade por parte dos estudantes com relação à compreensão conceitual, pois a maior parte do universo das teorias estudadas aborda fenômenos que ocorrem a nível microscópico e isto dificulta consideravelmente a aquisição da compreensão de conceitos essenciais à sua formação, uma vez que, neste nível, lhes falta o contato com informações sensoriais ${ }^{1-3}$.

Neste ponto pode-se destacar a química computacional, que hoje permite obter resultados altamente confiáveis de cálculos de propriedades, sendo aplicada com sucesso para estudo de uma ampla faixa de problemas de interesse químico, como cinética de reação (estados de transição) ${ }^{4}$, propriedades magnéticas (tensor blindagem e constante de acoplamento $)^{5}$, reatividade e estabilidade conformacional ${ }^{6}$. Vários destes estudos são baseados na teoria de FMO-Orbitais Moleculares de Fronteira (HOMO-LUMO $)^{4,7,8}$ e, mais recentemente, nas interações estereoeletrônicas hiperconjugativas ${ }^{9}$, através da análise dos Orbitais Naturais de Ligação $(\mathrm{NBO})^{10}$. Hoje, isto só é possível graças à melhoria das técnicas de cálculos e ao grande avanço na tecnologia dos computadores, o que resultou no desenvolvimento de métodos e programas especiais e de livre acesso, tanto para realização de cálculos como para visualização dos resultados.

Nos últimos anos temos trabalhado no estudo experimental e teórico de vários aspectos relacionados às reações de Diels-Alder de 2-ciclo-enonas com ciclopentadieno ${ }^{11,12}$. Por ex., em condições térmicas, dentre as 2-ciclo-enonas $\mathbf{2}-\mathbf{5}$ (Figura 1), apenas a nitrociclo-enona 2 reage com ciclopentadieno (1).

A reação de Diels-Alder é, no nosso entender, um excelente modelo para verificar a importância dos Orbitais de Fronteira (HOMO-LUMO) na avaliação da reatividade; além disso, a natureza elétron-captora dos substituintes ligados ao grupo $\mathrm{C}=\mathrm{C}$ das ciclo-enonas, pode ser utilizada para explicar os resultados encontra-

*e-mail: vljunior@usp.br
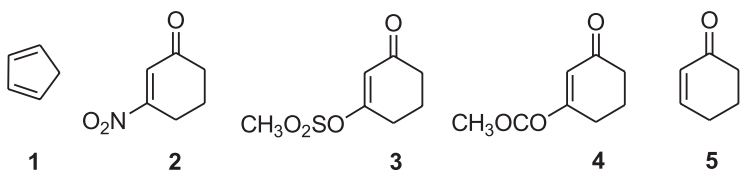

Figura 1.

dos pela diferença de energia $H O M O_{\text {dieno }}-L U M O_{\text {dienóflo }}$ e reafirmados pela análise dos Orbitais Naturais de Ligação.

Neste artigo é apresentada uma prática computacional na qual a reatividade em reações de Diels-Alder de 2-ciclo-enonas $\mathbf{2}$ - $\mathbf{5}$ com ciclopentadieno (1) é estudada por dois diferentes critérios: a diferença de energia entre os Orbitais Moleculares de Fronteira e as análises dos Orbitais Naturais de Ligação.

Esta prática computacional permite que os educadores consigam proporcionar condições aos alunos de, a partir da modelização do fenômeno estudado, desenvolver a compreensão conceitual dos temas abordados, não mais fazendo uso apenas mecânico dos conceitos que envolvem os fenômenos estudados. Este experimento é perfeitamente adequado para alunos do ensino superior em química e, por isso, os conceitos aqui envolvidos podem ser abordados nas aulas de química orgânica e físico-química, teóricas e experimentais.

\section{DESCRIÇÃO DO EXPERIMENTO}

Como preparação ao experimento computacional, o professor deve fornecer aos alunos algum embasamento teórico envolvendo a reação de Diels-Alder e as teorias de orbitais aqui utilizadas. Deve deixar claro alguns pontos fundamentais, como a influência da diferença de energia entre o orbital HOMO do dieno e o orbital LUMO do dienófilo (e vice-versa), reafirmando que quanto menor for esta diferença, mais fácil será a reação entre as duas espécies envolvidas.

Neste caso o professor deve expor um diagrama de orbitais como apresentado na Figura 2, mostrando que quando se combina um orbital ocupado com outro orbital desocupado, ocorre sempre uma certa diminuição de energia potencial, porque os dois elétrons podem ir ambos para o orbital ligante. Esta diminuição de energia, no entanto, é bem maior quando a diferença de energia entre $H O M O$ e $L U M O$ é pequena. 


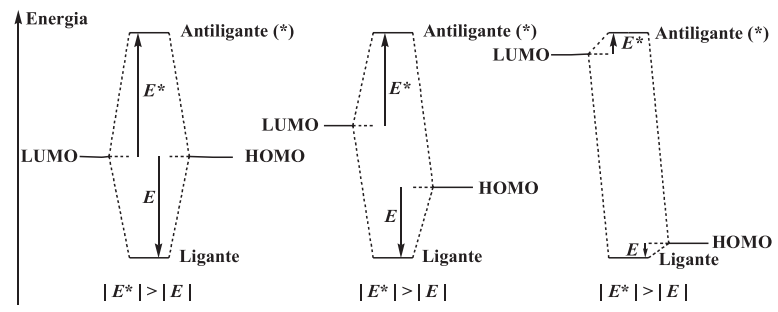

Figura 2.

Quando duas moléculas se aproximam para reagir, ocorre recombinação de todos os orbitais. A combinação de orbitais que contenham dois elétrons em cada um resulta em um aumento de energia potencial. Por isso, a diminuição de energia potencial pela combinação $H O M O+L U M O$ tem que ser superior ao aumento mencionado para que o produto tenha menor energia que os materiais de partida. É essencial, portanto, que a diferença de energia entre $H O M O$ e $L U M O$ seja relativamente pequena. Não podemos, porém, quantificar isto com facilidade; não é possível dizer a partir de qual valor de diferença entre HOMO e $L U M O$ vai ocorrer reação, porque não sabemos o valor exato do prejuízo causado pela combinação dos orbitais ocupados.

No caso dos Orbitais Naturais de Ligação (NBO), o professor deve ressaltar que este tipo de análise pode ser utilizada para avaliar os efeitos dos grupos substituintes ligados às espécies envolvidas na reação, indicando através de energias de estabilização (E) os efeitos doadores ou captores de elétrons que cada substituinte possui. Por exemplo, a interação entre o NBO ligante da dupla de um dienófilo com um NBO antiligante de um grupo captor de elétrons ligado a ele tem como resultado uma diminuição da densidade eletrônica na dupla dienofílica, tornando-a mais reativa. Este aumento da reatividade é provocado pelo abaixamento da energia de $L U M O$ do dienófilo, que é uma conseqüência direta da diminuição da densidade eletrônica, que faz com que os orbitais antiligantes do dienófilo se tornem mais propensos a adquirir cargas negativas.

A segunda parte da prática envolve o experimento computacional propriamente dito. Os alunos devem ser distribuídos em grupos e, sob a orientação do professor, construir as estruturas dos compostos $\mathbf{1}$ - $\mathbf{5}$ no programa Molden ${ }^{13,14}$. As geometrias são otimizadas (obtendo as estruturas de mínima energia) utilizando o programa Gaussian $98^{15,16}$ ou alternativamente o programa GAMESS ${ }^{17,18}$, que é de livre acesso. Nesta etapa do cálculo será utilizado o modelo teórico HF/STO-3G, utilizando-se PCs operando em sistema operacional Windows. As energias dos Orbitais de Fronteira HOMO-LUMO são calculadas com o modelo B3LYP/6$31+\mathrm{G}(\mathrm{d}, \mathrm{p})$ e as energias das interações entre os Orbitais Naturais de Ligação (NBO) em B3LYP/6-31G(d,p) no programa Gaussian 98. O programa MOLEKEL ${ }^{19,20}$ é utilizado para visualização das superfícies destes orbitais. Todos estes cálculos poderão ser feitos utilizando um único experimento, conforme exemplificado adiante (Figura 3), para a ciclo-enona 5. Este experimento foi planejado para ser concluído em uma sala de aula prática de $2 \mathrm{~h}$.

Como resultado, os alunos poderão visualizar a superfície dos Orbitais Moleculares de Fronteira e das interações dos Orbitais Naturais de Ligação e construir tabelas, gráficos ou figuras para facilitar a compreensão do estudo, como as apresentadas abaixo. O programa de visualização MOLEKEL permite realizar movimentos tridimensionais das estruturas, possibilitando ao aluno a observação de uma estrutura microscópica através de um modelo macroscópico.

\section{Análise dos orbitais moleculares de fronteira HOMO-LUMO}

Na Tabela 1 e Figura 4 são apresentadas as energias de HOMO$L U M O$ dos compostos $\mathbf{1}$ - 5, a modelização dos orbitais HOMO do

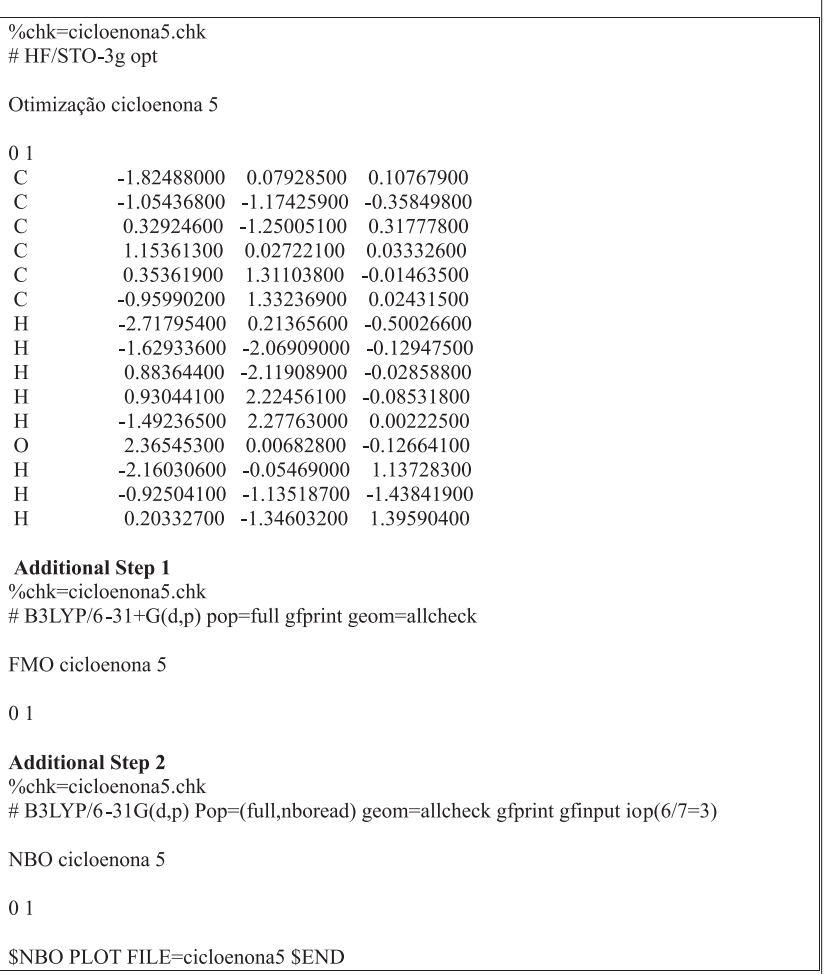

Figura 3.

dieno 1 e LUMO dos dienófilos $\mathbf{2}$ - $\mathbf{5}$ e suas respectivas diferenças de energia.

Estes resultados permitem aos alunos, além de visualizar a superfície dos orbitais moleculares, explicar por quê apenas a nitrociclo-enona 2 reage em condições térmicas com ciclopentadieno (é a que possui a menor diferença de energia entre HOMO-LUMO, $55,3 \mathrm{kcal} / \mathrm{mol}$ ), sendo que para as outras enonas $\mathbf{3}-\mathbf{5}$ esta diferença de energia é praticamente o dobro (97-106,7 kcal/mol). Esta diferença acentuada justifica qualitativamente o fato delas não regirem com o ciclopentadieno (1). Se considerarmos as diferenças de energia dos Orbitais de Fronteira a ordem de reatividade obtida é: $\mathbf{2}>>$ $\mathbf{3}>\mathbf{4}>\mathbf{5}$, o que está de acordo com a observação experimental, visto que em condições térmicas apenas a nitro-ciclo-enona 2 reage com o ciclopentadieno (1).

\section{Análise dos orbitais naturais de ligação (NBO)}

Na sua essência os NBOs podem ser descritos como orbitais moleculares localizados nas ligações químicas. Este conceito de

Tabela 1. Energias de HOMO e LUMO dos compostos $\mathbf{1}$ - 5 obtidos em B3LYP/6-31+G(d,p)

\begin{tabular}{lccc}
\hline Composto & $\mathrm{E}_{\text {номо }}(\mathrm{a} . \mathrm{u})$ & $\mathrm{E}_{\text {LUмо }}($ a.u $)$ & $\begin{array}{c}\Delta \mathrm{E}_{\text {LumoDień́filo-HoMoDieno }} \\
(\mathrm{kcal} / \mathrm{mol})^{*}\end{array}$ \\
\hline 1 & $-0,22921$ & $-0,02046$ & - \\
2 & $-0,28373$ & $-0,14109$ & 55,3 \\
3 & $-0,26390$ & $-0,07459$ & 97,0 \\
4 & $-0,25717$ & $-0,06332$ & 104,1 \\
5 & $-0,25153$ & $-0,05913$ & 106,7 \\
\hline
\end{tabular}

* para converter a diferença de energia $\Delta \mathrm{E}$ de a.u. para $\mathrm{kcal} / \mathrm{mol}$ basta multiplicá-la por 627,51. Por ex., para a combinação de $H O M O$ do composto 1 e $L U M O$ do composto $2 \Delta \mathrm{E}=-0,14109-(-0,22921)$ $=0,08812$ este valor multiplicado por $627,51=55,3 \mathrm{kcal} / \mathrm{mol}$ 


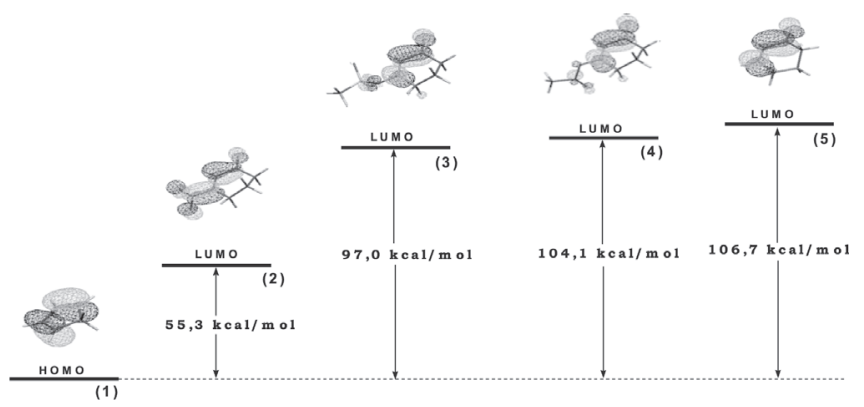

Figura 4.

orbitais naturais é usado para distribuir elétrons dentro de orbitais atômicos e moleculares, de modo a descrever cargas atômicas e ligações moleculares a partir da densidade eletrônica entre átomos. Desta maneira, é possível avaliar quantitativamente o efeito elétron-captor exercido pelos grupos ligados à ligação $\mathrm{C}=\mathrm{C}$ das ciclo-enonas $\mathbf{2}-\mathbf{5}$, através da interação entre o orbital $\pi$ ligante da ligação $\mathrm{C}=\mathrm{C}$ e os orbitais antiligantes dos grupos carbonila e dos substituintes $\left(\mathrm{NO}_{2}, \mathrm{OCOCH}_{3}\right.$ e $\left.\mathrm{OSO}_{2} \mathrm{CH}_{3}\right)$. Na Tabela 2 e Figura 5 são apresentadas, respectivamente, as interações significantes entre orbitais-NBOs e visualizações

Tabela 2. Interações significantes entre orbitais-NBO para os compostos $\mathbf{2}$ - $\mathbf{5}$ obtidos em B3LYP/6-31G(d,p)

\begin{tabular}{lccc}
\hline Composto & Interação-NBO & $\mathrm{E}(\mathrm{kcal} / \mathrm{mol})$ & $\mathrm{E}($ total) $(\mathrm{kcal} / \mathrm{mol})$ \\
\hline $\mathbf{2}$ & $\pi_{\mathrm{C}=\mathrm{C}} \rightarrow \pi^{*}{ }_{\mathrm{N}=\mathrm{O}}$ & 17,83 & 32,10 \\
& $\pi_{\mathrm{C}=\mathrm{C}} \rightarrow \pi^{*}{ }_{\mathrm{C}=\mathrm{O}}$ & 14,27 & \\
$\mathbf{3}$ & $\pi_{\mathrm{C}=\mathrm{C}} \rightarrow \sigma^{*}{ }^{*}{ }_{\mathrm{O}-\mathrm{S}}$ & 3,43 & 20,16 \\
$\mathbf{4}$ & $\pi_{\mathrm{C}=\mathrm{C}} \rightarrow \pi^{*}{ }_{\mathrm{C}=\mathrm{O}}$ & 16,73 & \\
& $\pi_{\mathrm{C}=\mathrm{C}} \rightarrow \sigma^{*}{ }^{\mathrm{O}-\mathrm{C}}$ & 1,58 & 19,06 \\
$\mathbf{5}$ & $\pi_{\mathrm{C}=\mathrm{C}} \rightarrow \pi^{*}{ }_{\mathrm{C}=\mathrm{O}}$ & 17,48 & \\
\hline
\end{tabular}

A análise dos NBOs (Tabela 2 e Figura 5) mostra claramente a natureza elétron-captora dos grupos $\mathrm{C}=\mathrm{O}$ e dos substituintes $\left(\mathrm{NO}_{2}\right.$, $\mathrm{OCOCH}_{3}$ e $\mathrm{OSO}_{2} \mathrm{CH}_{3}$ ) na ligação $\mathrm{C}=\mathrm{C}$ das ciclo-enonas $\mathbf{2}-\mathbf{5}$, indicando que quanto maior for o efeito elétron-captor destes grupos, menor é a densidade eletrônica na ligação $\mathrm{C}=\mathrm{C}$ dos dienófilos. A diminuição desta densidade eletrônica em $\mathrm{C}=\mathrm{C}$ provoca uma diminuição da energia do orbital LUMO das ciclo-enonas, tornando-as mais reativas. Se considerarmos a soma dos efeitos elétron-captores $\left(\mathrm{E}_{\text {(total }}\right)$ a ordem de reatividade fornecida pela análise dos NBOs também é: $\mathbf{2}>\mathbf{3}>\mathbf{4}>\mathbf{5}$.

\section{CONCLUSÕES}

Nesta prática computacional, é apresentada uma boa aplicação do conceito de Orbitais Moleculares de Fronteira (HOMO-LUMO) utilizando algumas reações de Diels-Alder de 2-ciclo-enonas com ciclopentadieno. Foram avaliadas também as influências dos grupos $\mathrm{C}=\mathrm{O}$ e dos substituintes $\left(\mathrm{NO}_{2}, \mathrm{OCOCH}_{3}\right.$ e $\left.\mathrm{OSO}_{2} \mathrm{CH}_{3}\right)$, ligados à ligação $\mathrm{C}=\mathrm{C}$ das ciclo-enonas através dos Orbitais Naturais de Ligação (NBOs).

As duas teorias aplicadas permitem estabelecer não somente uma ordem de reatividade das ciclo-enonas mas, também, explicar porque apenas a nitro-ciclo-enona $\mathbf{2}$ reage em condições térmicas com o dieno $\mathbf{1}$.
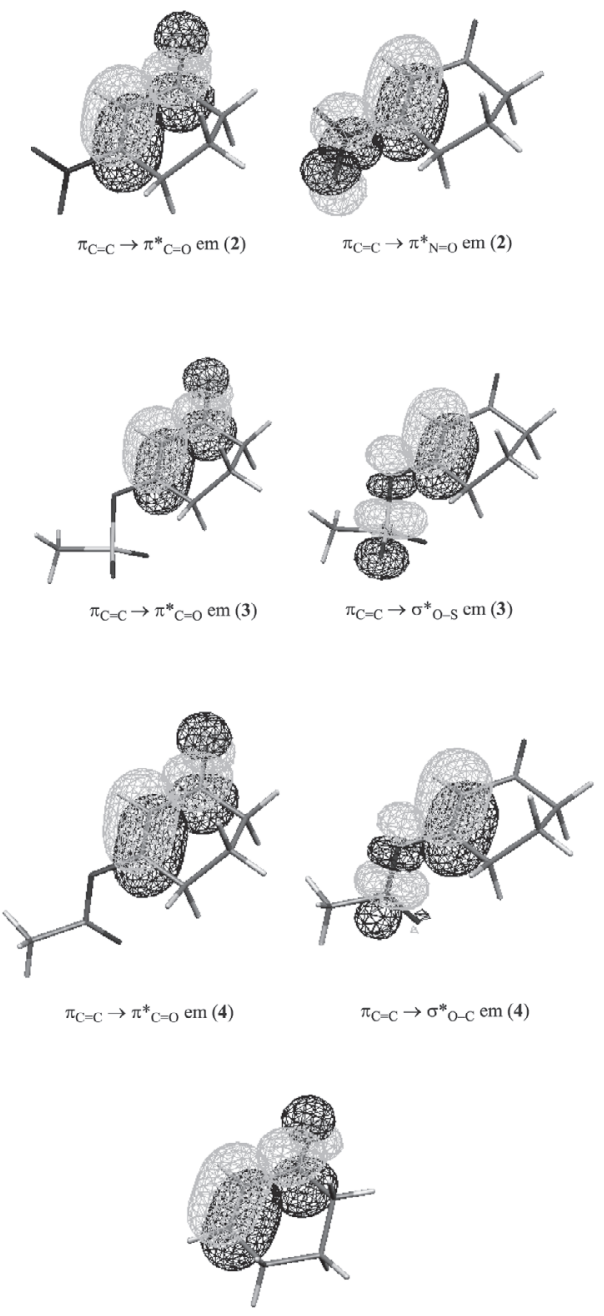

$\pi_{\mathrm{C}-\mathrm{C}} \rightarrow \pi^{*}{ }_{\mathrm{C}-\mathrm{O}} \mathrm{em}(\mathbf{5})$

Figura 5.

Com este experimento os alunos podem também visualizar as estruturas e as superfícies dos orbitais sem a necessidade de recursos computacionais avançados.

\section{AGRADECIMENTOS}

À Fundação de Amparo à Pesquisa do Estado de São Paulo (FAPESP), ao Conselho Nacional de Desenvolvimento Científico e Tecnológico (CNPq), à Coordenadoria de Aperfeiçoamento de Pessoal do Nível Superior (CAPES) e à Financiadora de Estudos e Projetos (FINEP) pelo suporte financeiro.

\section{REFERÊNCIAS E NOTAS}

1. Bunge, M.; Ciência e Desenvolvimento, Itatiaia: Belo Horizonte, USP, São Paulo, 1989, p. 32

2. Wu, H.; Krajcik, J. S.; Soloway, E.; J. Res. Sci. Teaching 2001, 38, 821

3. Ribeiro, A. A.; Greca, I. M.; Quim. Nova 2003, 26, 542.

4. Domingo, L. R.; Aurell, M. J.; Pérez, P.; Contreras, R.; J. Org. Chem. 2003, 68,3884 .

5. Oliveira, K. T.; Lacerda Jr., V.; Constantino, M. G.; Donate, P. M.; da Silva, G. V. J.; Brocksom, T. J.; Frederico, D.; Spectrochim. Acta, Part A 2006, 63, 709.

6. Weinhold, F.; Nature 2001, 411, 539; Pophristic, V.; Goodman L.; Nature 2001, 411, 565 .

7. Galembeck, S. E.; Caramori, G. F.; Quim. Nova 2003, 26, 957.

8. Fleming, I.; Frontier Orbitals and Organic Chemical Reactions, John Wiley and Sons: New York, 1976. 
9. Schreiner, P. R.; Angew. Chem., Int. Ed. 2002, 41, 3579.

10. Foster, J. P.; Weinhold, F.; J. Am. Chem. Soc. 1980, 102, 7211; Weinhold, F.; Landis, C.; Valency and Bonding: A Natural Bond Orbital DonorAcceptor Perpective, Cambridge University Press: Cambridge, 2005.

11. da Silva Filho, L. C.; Lacerda Jr., V.; Constantino, M. G.; Invernize, P. R.; da Silva, G. V. J.; Beilstein J. Org. Chem. 2005, 1, 14

12. Lacerda Jr., V.; Oliveira, K. T.; da Silva Filho, L. C.; Constantino, M. G.; Galembeck, S. E.; Resumos do XII Simpósio Brasileiro de Química Teórica, Caxambu, Brasil, 2003.

13. Schaftenaar, G.; Noordik, J. H.; J. Comput.-Aided Mol. Design 2000, 14, 123; http://www.cmbi.ru.nl/molden/windowsnt95.html\#install, acessada em Maio 2005.

14. Este programa é de livre acesso, no entanto, oferece algumas dificuldades de uso ou requer alguma dedicação para construir as estruturas. Alternativamente podem ser utilizados outros programas como o próprio Gauss View da Gaussian ou PCModel, que são de baixo custo e fácil uso. Para construir as estruturas no Molden copie o programa, que pode ser obtido no endereço acima, e não se esqueça de instalar um modulador para Windows (MI-X4.2) também disponível no mesmo endereço. Inicialmente ative o MIX4.2 (abra o programa) e, em seguida, abra o Molden.exe. Note que o programa Molden não precisa ser instalado, apenas deve ser copiado em um diretório do PC. Em seguida selecione o comando "Add Line" e escolha os átomos, adicionando-os sucessivamente. Há também fragmentos de anel que podem ser prontamente selecionados. Caso necessário, os comprimentos de ligação e ângulos podem ser ajustados manualmente. Após desenhar, escolha o formato em que o arquivo será lido. Por ex., se os cálculos serão efetuados no Gaussian escrever: (nomedoarquivo.com) e salvar selecionando o comando "Write-ZMatrix". O arquivo será salvo no diretório do programa. Maiores detalhes podem ser obtidos no site do programa.

15. Frisch, M. J.; Trucks, G. W.; Schlegel, H. B.; Scuseria, G. E.; Robb, M. A.; Cheeseman, J. R.; Montgomery, Jr., J. A.; Vreven, T.; Kudin, K. N.; Burant, J. C.; Millam, J. M.; Iyengar, S. S.; Tomasi, J.; Barone, V.; Mennucci, B.; Cossi, M.; Scalmani, G.; Rega, N.; Petersson, G. A.; Nakatsuji, H.; Hada, M.; Ehara, M.; Toyota, K.; Fukuda, R.; Hasegawa, J.; Ishida, M.; Nakajima, T.; Honda, Y.; Kitao, O.; Nakai, H.; Klene, M.; Li, X.; Knox, J. E.; Hratchian, H. P.; Cross, J. B.; Bakken, V.; Adamo, C.;
Jaramillo, J.; Gomperts, R.; Stratmann, R. E.; Yazyev, O.; Austin, A. J.; Cammi, R.; Pomelli, C.; Ochterski, J. W.; Ayala, P. Y.; Morokuma, K.; Voth, G. A.; Salvador, P.; Dannenberg, J. J.; Zakrzewski, V. G.; Dapprich, S.; Daniels, A. D.; Strain, M. C.; Farkas, O.; Malick, D. K.; Rabuck, A. D.; Raghavachari, K.; Foresman, J. B.; Ortiz, J. V.; Cui, Q.; Baboul, A. G.; Clifford, S.; Cioslowski, J.; Stefanov, B. B.; Liu, G.; Liashenko, A.; Piskorz, P.; Komaromi, I.; Martin, R. L.; Fox, D. J.; Keith, T.; Al-Laham, M. A.; Peng, C. Y.; Nanayakkara, A.; Challacombe, M.; Gill, P. M. W.; Johnson, B.; Chen, W.; Wong, M. W.; Gonzalez, C.; Pople, J. A.; Gaussian 98, Revision A.6; Gaussian, Inc., Pittsburgh, 1998.

16. Para submeter os cálculos, abra o programa Gaussian, clique "File" e depois "Open". Procure o arquivo gerado no Molden ou programas equivalentes e solicite "Abrir". Este arquivo irá conter as coordenadas dos átomos. Adicione as linhas de comando conforme modelo apresentado na Figura 3. Em seguida selecione o comando "Run"; logo após será perguntado em qual diretório o resultado será salvo (nomedoarquivo.out) selecione o local e mande "Salvar".

17. Schmidt, M. W.; Baldridg, K. K.; Boatz, J. A.; Elbert, S. T.; Gordon, M S.; Jensen, J. H.; Kosek, S.; Matsunaga, N.; Nguyen, K. A.; Su, S. J.; Windus, T. L.; Dupuis, M.; Montgomery, J. A.; J. Comput. Chem. 1993, 14, 1347.

18. Este programa pode ser obtido livremente na página: http:// www.msg.ameslab.gov/GAMESS/pcgamess.shtml. Sua utilização é semelhante ao Gaussian, no entanto, para realização do cálculos de NBO é necessário adquirir o software "NBO" separadamente. No programa Gaussian este software já vem implementado.

19. Plükiger, P.; Lüthi, H. P.; Portmann, S.; Weber, J.; MOLEKEL 4.1, Swiss Center for Scientific Computing, Switzerland, 2000-2001.

20. Este programa pode ser obtido na página http://www.cscs.ch/molekel/. Para visualizar as estruturas abra o programa (molekel.exe) e com o botão direito do mouse selecione a opção"Load" e depois "gaussian log". Para visualizar os orbitais de fronteira (FMO) selecionar a opção "Compute" e depois "Orbital". O processo de visualização dos NBOs é um pouco mais trabalhoso. Inicialmente abra o arquivo que contenha a estrutura. Depois abra na opção "NBO orb" o arquivo com extensão (.37) gerado pelo cálculo na pasta "Scratch" do diretório G98. Selecione as interações e gere as superfícies. Maiores detalhes podem ser obtidos no site do programa. 